\title{
Influence of the Coulomb potential on above-threshold ionization: a quantum-orbit analysis beyond the strong-field approximation
}

\author{
X.-Y. Lai ${ }^{1,2}$, C. Poli ${ }^{3}$, H. Schomerus ${ }^{3}$ and C. Figueira de Morisson Faria ${ }^{1}$ \\ ${ }^{1}$ Department of Physics and Astronomy, University College London, \\ Gower Street, London WC1E 6BT, United Kingdom \\ ${ }^{2}$ State Key Laboratory of Magnetic Resonance and Atomic and Molecular Physics, \\ Wuhan Institute of Physics and Mathematics, Chinese Academy of Sciences, Wuhan 430071, China \\ ${ }^{3}$ Department of Physics, Lancaster University, LA1 4YB Lancaster, United Kingdom
}

(Dated: August 11, 2015)

\begin{abstract}
We perform a detailed analysis of how the interplay between the residual binding potential and a strong laser field influences above-threshold ionization (ATI), employing a semi-analytical, Coulombcorrected strong-field approximation (SFA) in which the Coulomb potential is incorporated in the electron propagation in the continuum. We find that the Coulomb interaction lifts the degeneracy of some SFA trajectories, and we identify a set of orbits which, for high enough photoelectron energies, may be associated with rescattering. Furthermore, by performing a direct comparison with the standard SFA, we show that several features in the ATI spectra can be traced back to the influence of the Coulomb potential on different electron trajectories. These features include a decrease in the contrast, a shift towards lower energies in the interference substructure, and an overall increase in the photoelectron yield. All features encountered exhibit a very good agreement with the $a b$ initio solution of the time-dependent Schrödinger equation.
\end{abstract}

PACS numbers: $33.80 . \mathrm{Rv}, 33.80 . \mathrm{Wz}, 42.50 . \mathrm{Hz}$

\section{INTRODUCTION}

When matter interacts with a strong laser field of peak intensity around $10^{14} \mathrm{~W} / \mathrm{cm}^{2}$, the outmost electron may be freed by absorbing many more photons than necessary. This very highly nonlinear process is known as abovethreshold ionization (ATI) and has attracted considerable attention since the early work of Agostini and coworkers [1]; for a review see Ref. [2]. For typical parameters employed in experiments, i.e., near infra-red laser fields, it is commonly accepted that the electron reaches the continuum by tunnel ionization. If the released electron revisits the parent ion in the presence of the laser field $[3,4]$, this results in various additional highly nonlinear phenomena, such as high-order ATI (HATI) [5], highorder harmonic generation (HHG) [6], and nonsequential double ionization (NSDI) [7]. Recently, considerable progress has been made in the study of these nonlinear strong-field phenomena. For example, both ATI and HHG have been employed as an important technique to explore the electron shell structure and sub-femtosecond dynamics [8-12], and NDSI has opened the door to the study of strong-field electron-electron correlation [13-16].

In order to uncover the underlying physics of these highly nonlinear phenomena, many theories and models have been proposed, such as the $a b$ initio solution of the time-dependent Schrödinger equation (TDSE) [17] and the quantum-orbit theory within the strong-field approximation (SFA) [18-20]. Since the TDSE contains no physical approximation, its outcome is widely taken as a benchmark to evaluate the data in experiments and the calculations of other theories and models [21-23]. However, in many occasions the TDSE does not provide a transparent physical picture. Furthermore, since the numerical effort involved in ab initio computations increases exponentially with the degrees of freedom, its implementation is impractical for strongly correlated multielectron systems. In contrast, the quantum-orbit theory provides very clear physical insight in terms of distinct electron ionization trajectories, and its outcome is qualitatively consistent with the experimental data. Therefore, it has been widely and successfully used in the modeling of strong-field phenomena [18, 24-26]

One should note, however, that the validity of the conventional quantum-orbit theory is limited. In fact, the use of the SFA before the application of the saddlepoint approximation implies that a considerable amount of physics is left out for the sake of a clear and intuitive picture [2]. In particular, the SFA fully neglects the effect of the Coulomb potential of the parent ion on the ionized electrons, approximating the continuum states by field-dressed plane waves [27]. For single charged negative ions, this approximation is justified as the Coulomb interaction between the neutral core and the freed electron is absent. However, for atoms and molecules, this interaction is present, so that the SFA only works qualitatively. Furthermore, in recent years, several features have been observed which clearly highlight the influence of the Coulomb potential. Examples are the so-called low-energy structure (LES) in ATI spectra [28-30], fanshaped structures in photoelectron momentum distributions [31-35], and the violation of the fourfold symmetry in angular electron distributions for elliptically polarized fields [36, 37].

Motivated by these observations, many methods have been developed in the past few years in order to account for the Coulomb potential in orbit-based methods. These include (i) using Coulomb-Volkov functions to describe the electron continuum states in the SFA [38-40]; (ii) incorporating the binding potential in the electron propagation using the eikonal Volkov approximation [41, 42]; 
(iii) a Coulomb-corrected SFA (CCSFA), which takes the trajectories from the SFA theory as a zero-order approximation and accounts for the Coulomb field perturbatively [35, 43, 44]; (iv) a quantum-trajectory Monte Carlo (QTMC), which is based on Classical-Trajectory MonteCarlo (CTMC) simulations, but considers the phase of each trajectory [45]; (v) initial-value representations such as the Herman-Kluk propagator [46-49] and the Coupled Coherent States method [50-52]; (vi) a time-dependent analytical $R$-matrix approach [53], which divides the space into inner and outer regions and has been successfully used to study the many-electron systems [54].

Most of the above-mentioned approaches have been applied to and tested on direct ATI. This phenomenon is a particularly good testing ground for Coulomb corrections for two main reasons. First, the momentum range involved is relatively low, so that the influence of the Coulomb potential is expected to be significant. Second, in contrast to high-order ATI, hard collisions are expected to be absent, so that the Coulomb corrections are in principle easier to implement. In particular the influence of the Coulomb potential on quantum-interference patterns has attracted a great deal of attention [35]. Furthermore, it has been shown that the presence of the Coulomb potential considerably alters the topology of the orbits, giving rise to types of trajectories that are absent in the SFA [44]. In particular, in Ref. [35] it has been shown that sub-barrier corrections are necessary in order to obtain the correct phases in the ATI electron momentum distributions.

In this work, we develop a quantum-orbit theory with Coulomb interactions that, besides the effect on the phase, also accounts for the influence of the interactions on the semiclassical amplitudes. We find that the amplitude is significantly modified both via the atomic dipole moment at ionization and due to the altered stability of the trajectories during the electron propagation through the continuum. This Coulomb-corrected method is then employed to study the influence of the Coulomb potential on the direct ATI ionization spectrum of Hydrogen. We perform a systematic investigation of how the Coulomb coupling changes the topology of the trajectories, which are either decelerated or accelerated with regard to their SFA counterparts. This leads to a decrease in the phase difference between the contributions from different types of trajectories, which influences the interference patterns in the spectra. We also discuss how momentum nonconservation lifts the degeneracy of certain SFA trajectories. Furthermore, we verify that the distinction between direct and rescattered trajectories is blurred by the presence of the binding potential, which causes a set of trajectories to go around the core.

Our results show that the spectrum calculated with this method is in much better agreement with the $a b$ initio TDSE result than the predictions of the standard SFA. In particular, the Coulomb-corrected theory recovers the much weaker contrast in the interference substructure observed in the TDSE, and relates this effect to the unequal semiclassical weights of the electron trajectories in the presence of the Coulomb interactions. Similarly to what has been encountered in [35], we also observe that the positions of the interference maxima in the spectrum from the quantum-orbit theory and TDSE result are shifted with respect to the SFA simulations. However, our model indicates that these shifts mainly stem from the modified electron propagation in the continuum.

This article is organized as follows. In Sec. II we describe the theoretical models employed in this paper, namely the standard SFA and the SFA with Coulomb corrections, starting from the TDSE. In Sec. III we apply the theories to direct ATI and discuss the consequences of the Coulomb interactions, first in terms of the individual trajectories and then for the resulting ionization spectrum. Finally, in Sec. IV, we summarize the main conclusions to be drawn from this work. We use atomic units throughout.

\section{THEORETICAL MODELS}

The underlying framework for the subsequent discussions is the time-dependent Schrödinger equation

$$
i \partial_{t}|\psi(t)\rangle=H(t)|\psi(t)\rangle
$$

In the ionization problems considered in this work, the Hamiltonian separates into two parts, $H(t)=H_{a}+H_{I}(t)$. Here

$$
H_{a}=\frac{\hat{\mathbf{p}}^{2}}{2}+V(\hat{\mathbf{r}})
$$

denotes the field-free one-electron atomic Hamiltonian and the hats denote operators. In the problem addressed here, we consider a Coulomb-type potential

$$
V(\hat{\mathbf{r}})=-\frac{C}{\sqrt{\hat{\mathbf{r}} \cdot \hat{\mathbf{r}}}},
$$

where $0 \leq C \leq 1$ is an effective coupling, which we vary in a continuous fashion in order to assess the influence of the Coulomb potential. For Hydrogen, $C=1$. Furthermore, $H_{I}(t)$ describes the interaction with the laser field. In the velocity and length gauges, this interaction is given by $H_{I}(t)=\hat{\mathbf{p}} \cdot \mathbf{A}(t)+\mathbf{A}^{2} / 2$ and $H_{I}(t)=-\hat{\mathbf{r}} \cdot \mathbf{E}(t)$, respectively, where $\mathbf{E}(t)=-d \mathbf{A}(t) / d t$ is the external laser field. The length gauge provides us with the physical picture of ionization as a tunneling process driven by an effective time-dependent potential. This gauge will be employed throughout.

The time-evolution operator associated with this Hamiltonian is of the general form

$$
U\left(t, t_{0}\right)=\mathcal{T} \exp \left[i \int_{t_{0}}^{t} H\left(t^{\prime}\right) d t^{\prime}\right],
$$

where $\mathcal{T}$ denotes time-ordering. This operator takes a wave function from a time $t_{0}$ to a time $t$, i.e., $|\psi(t)\rangle=$ $U\left(t, t_{0}\right)\left|\psi\left(t_{0}\right)\right\rangle$, and satisfies

$$
\begin{aligned}
i \partial_{t} U\left(t, t_{0}\right) & =H(t) U\left(t, t_{0}\right), \\
-i \partial_{t_{0}} U\left(t, t_{0}\right) & =U\left(t, t_{0}\right) H\left(t_{0}\right) .
\end{aligned}
$$


Employing the Dyson equation, the time-evolution operator may be written as

$$
U\left(t, t_{0}\right)=U_{a}\left(t, t_{0}\right)-i \int_{t_{0}}^{t} U\left(t, t^{\prime}\right) H_{I}\left(t^{\prime}\right) U_{a}\left(t^{\prime}, t_{0}\right) d t^{\prime},
$$

where $U_{a}\left(t, t_{0}\right)$ is the time-evolution operator associated with the field-free Hamiltonian.

For above-threshold ionization, the initial state is a bound state $\left|\psi_{0}\right\rangle$, while the final state is a continuum state $\left|\psi_{\mathbf{p}}(t)\right\rangle$ with drift momentum p. This gives the ionization amplitude [2]

$$
M(\mathbf{p})=-i \lim _{t \rightarrow \infty} \int_{-\infty}^{t} d t^{\prime}\left\langle\psi_{\mathbf{p}}(t)\left|U\left(t, t^{\prime}\right) H_{I}\left(t^{\prime}\right)\right| \psi_{0}\left(t^{\prime}\right)\right\rangle
$$

which is formally exact.

\section{A. Strong-field approximation}

Equation (7) cannot be solved in closed form, so that approximations are required in order to compute the ATI transition amplitude via analytical methods. A popular approximation is to replace $U\left(t, t^{\prime}\right)$ by the Volkov time evolution operator $U^{(V)}\left(t, t^{\prime}\right)$ in Eq. (7). This implies that the continuum has been approximated by Volkov states, i.e., by field-dressed plane waves $\left|\psi_{\mathbf{p}}^{(V)}(t)\right\rangle$, where

$$
\left\langle\mathbf{r} \mid \psi_{\mathbf{p}}^{(V)}(t)\right\rangle=\langle\mathbf{r} \mid \tilde{\mathbf{p}}(t)\rangle \exp \left[-i \int_{-\infty}^{t} d \tau \frac{[\mathbf{p}+\mathbf{A}(\tau)]^{2}}{2}\right]
$$

with

$$
\langle\mathbf{r} \mid \tilde{\mathbf{p}}(t)\rangle=\frac{\exp [i \tilde{\mathbf{p}}(t) \cdot \mathbf{r}]}{(2 \pi)^{3 / 2}} .
$$

Here $\tilde{\mathbf{p}}(t)=\mathbf{p}+\mathbf{A}(t)$ in the length gauge and $\tilde{\mathbf{p}}(t)=\mathbf{p}$ in the velocity gauge, so that $U^{(V)}\left(t, t^{\prime}\right)\left|\psi_{\mathbf{p}}\left(t^{\prime}\right)\right\rangle=\left|\psi_{\mathbf{p}}^{(V)}(t)\right\rangle$ [? ]. This is the key idea behind the strong-field approximation or Keldysh-Faisal-Reiss theory [27, 55, 56]. For detailed discussions see, e.g., $[57,58]$ and the recent tutorials [59]. Within the SFA, the amplitude (7) is then given by $[2,27,55,56]$

$$
M(\mathbf{p})=-i \int_{-\infty}^{\infty} d t^{\prime}\left\langle\mathbf{p}+\mathbf{A}\left(t^{\prime}\right)\left|H_{I}\left(t^{\prime}\right)\right| \psi_{0}\right\rangle e^{i S\left(\mathbf{p}, t^{\prime}\right)} .
$$

Here

$$
S\left(\mathbf{p}, t^{\prime}\right)=-\frac{1}{2} \int_{t^{\prime}}^{\infty}[\mathbf{p}+\mathbf{A}(\tau)]^{2} d \tau+I_{p} t^{\prime}
$$

is the semiclassical action, where $I_{p}$ gives the ionization potential and $\mathbf{A}(t)$ denotes the vector potential of the laser field. In Eq. (10), we have also employed the notation $\left|\psi_{0}\left(t^{\prime}\right)\right\rangle=e^{i I_{p} t^{\prime}}\left|\psi_{0}\right\rangle$.

For sufficiently high intensity and low frequency of the laser field, the temporal integration in Eq. (10) can be evaluated by the saddle-point method [19, 20], which seeks solutions such that the action (11) is stationary. The corresponding saddle-point equation reads

$$
\frac{\left[\mathbf{p}+\mathbf{A}\left(t^{\prime}\right)\right]^{2}}{2}+I_{p}=0
$$

Physically, Eq. (12) ensures the conservation of energy at the ionization time $t^{\prime}$, which leads to complex solutions $t_{s}$. In terms of these solutions, the transition amplitude (10) can then be written as

$$
M(\mathbf{p}) \sim \sum_{s} \mathcal{C}\left(t_{s}\right)\left\langle\mathbf{p}+\mathbf{A}\left(t_{s}\right)\left|H_{I}\left(t_{s}\right)\right| \psi_{0}\right\rangle e^{i S\left(\mathbf{p}, t_{s}\right)}
$$

where the prefactors

$$
\mathcal{C}\left(t_{s}\right)=\sqrt{\frac{2 \pi i}{\partial^{2} S\left(\mathbf{p}, t_{s}\right) / \partial t_{s}^{2}}}
$$

are expected to vary much more slowly than the action for the saddle-point approximation to hold. Since each solution $t_{s}$ represents a distinct trajectory of the electron in the laser field, the sum in Eq. (13) denotes the interference between different quantum paths, which has been extensively studied in the literature (for reviews see, e.g., $[2,60])$. One should note that in the SFA, the fielddressed momentum is conserved.

\section{B. Coulomb Quantum-orbit Strong-Field Approximation}

Within the SFA, an electron no longer feels the atomic potential after it has been promoted into the continuum at the time $t_{s}$, resulting in the considerable deviations between this model and experimental results. In this section we describe a Coulomb Quantum orbit Strong-Field Approximation (CQSFA) which cures this shortcoming of the SFA (for similar approaches see [37, 41-43]).

\section{General considerations and derivation}

In the presence of the Coulomb potential,the fielddressed momentum is no longer conserved, and the time evolution operator depends on both $\hat{\mathbf{r}}$ and $\hat{\mathbf{p}}$. As a result, the time evolution operator cannot be diagonalized by the Volkov states (8). to compute the transition amplitude (7). First, it is useful to introduce the closure relation $\int d \tilde{\mathbf{p}}_{0}\left|\tilde{\mathbf{p}}_{0}\right\rangle\left\langle\tilde{\mathbf{p}}_{0}\right|=1$, so that Eq. (7) is rewritten as

$$
M\left(\mathbf{p}_{f}\right)=-i \lim _{t \rightarrow \infty} \int_{-\infty}^{t} d t^{\prime} \int d \tilde{\mathbf{p}}_{0}\left\langle\tilde{\mathbf{p}}_{f}(t)\left|U\left(t, t^{\prime}\right)\right| \tilde{\mathbf{p}}_{0}\right\rangle\left\langle\tilde{\mathbf{p}}_{0}\left|H_{I}\left(t^{\prime}\right)\right| \psi_{0}\left(t^{\prime}\right)\right\rangle
$$

where $\left|\tilde{\mathbf{p}}_{f}(t)\right\rangle=\left|\psi_{\mathbf{p}}(t)\right\rangle$ and $\tilde{\mathbf{p}}_{0}=\mathbf{p}_{0}+\mathbf{A}\left(t^{\prime}\right)$ denotes the velocity of the electron at the initial time $t^{\prime}$. Similarly, the final electron velocity is given by $\tilde{\mathbf{p}}_{f}(t)=\mathbf{p}_{f}+\mathbf{A}(t)$.

The momentum-space matrix element $\left\langle\tilde{\mathbf{p}}_{f}(t)\left|U\left(t, t^{\prime}\right)\right| \tilde{\mathbf{p}}_{0}\right\rangle$ will now be rewritten by employing the Feynman path-integral formalism [65]. This 
means that the time-evolution operator $U\left(t, t^{\prime}\right)$ is sliced into $N+1$ time-evolution operators with an infinitesimal time slice of width $\varepsilon=t_{n}-t_{n-1}=\left(t-t^{\prime}\right) /(N+1)$, where $\tilde{\mathbf{p}}_{f}(t)=\tilde{\mathbf{p}}_{N+1}, t_{N+1}=t$, and $t_{0}=t^{\prime}$. This gives

$$
\left\langle\tilde{\mathbf{p}}_{f}(t)\left|U\left(t, t^{\prime}\right)\right| \tilde{\mathbf{p}}_{0}\right\rangle=\prod_{n=1}^{N}\left[\int d \tilde{\mathbf{p}}_{n}\right] \prod_{n=1}^{N+1}\left[\int \frac{d \mathbf{r}_{n}}{(2 \pi)^{3}}\right] e^{i \mathcal{A}_{N}},
$$

where

$\mathcal{A}_{N}=\sum_{n=1}^{N+1}-\left(\tilde{\mathbf{p}}_{n}-\tilde{\mathbf{p}}_{n-1}\right) \cdot \mathbf{r}_{n}-\varepsilon\left[\tilde{\mathbf{p}}_{n}^{2} / 2+\mathbf{r}_{n} \cdot \mathbf{E}\left(t_{n}\right)+V\left(\mathbf{r}_{n}\right)\right]$.

In the continuous limit,

$$
\left\langle\tilde{\mathbf{p}}_{f}(t)\left|U\left(t, t^{\prime}\right)\right| \tilde{\mathbf{p}}_{0}\right\rangle=\int_{\tilde{\mathbf{p}}_{0}}^{\tilde{\mathbf{p}}_{\mathbf{f}}(\mathbf{t})} \mathcal{D}^{\prime} \tilde{\mathbf{p}} \int \frac{\mathcal{D} \mathbf{r}}{(2 \pi)^{3}} e^{i \mathcal{A}(\tilde{\mathbf{p}}, \mathbf{r})}
$$

with

$$
\mathcal{A}(\tilde{\mathbf{p}}, \mathbf{r})=\int_{t^{\prime}}^{t} d \tau[-\dot{\tilde{\mathbf{p}}} \cdot \mathbf{r}(\tau)-H(\tilde{\mathbf{p}}, \mathbf{r}, \tau)]
$$

and

$$
H(\tilde{\mathbf{p}}, \mathbf{r}, \tau)=\tilde{\mathbf{p}}^{2} / 2+\mathbf{r}(\tau) \cdot \mathbf{E}(\tau)-\frac{C}{\sqrt{\mathbf{r}(\tau) \cdot \mathbf{r}(\tau)}},
$$

where $\tilde{\mathbf{p}}(\tau)=\mathbf{p}+\mathbf{A}(\tau), t^{\prime} \leq \tau \leq t$, is the intermediate velocity of the electron and $\mathcal{D}^{\prime}$ expresses the fact that there are fewer integrals in $\mathbf{p}_{n}$ [see Eq. (16)]. Physically, Eq. (18) denotes the integration over all paths from $\tilde{\mathbf{p}}_{0}$ to $\tilde{\mathbf{p}}_{f}(t)$.

Therefore, the Coulomb corrected transition amplitude becomes

$$
\begin{aligned}
M\left(\mathbf{p}_{f}\right)= & -i \lim _{t \rightarrow \infty} \int_{-\infty}^{t} d t^{\prime} \int d \tilde{\mathbf{p}}_{0} \int_{\tilde{\mathbf{p}}_{0}}^{\tilde{\mathbf{p}}_{f}(t)} \mathcal{D}^{\prime} \tilde{\mathbf{p}} \int \frac{\mathcal{D} \mathbf{r}}{(2 \pi)^{3}} \\
& \times e^{i S\left(\tilde{\mathbf{p}}, \mathbf{r}, t^{\prime}, t\right)}\left\langle\tilde{\mathbf{p}}_{0}\left|H_{I}\left(t^{\prime}\right)\right| \psi_{0}\right\rangle
\end{aligned}
$$

where the action is given by

$$
S\left(\tilde{\mathbf{p}}, \mathbf{r}, t^{\prime}, t\right)=I_{p} t^{\prime}+\mathcal{A}(\tilde{\mathbf{p}}, \mathbf{r}),
$$

with $\mathbf{E}(t)=-\partial_{t} \mathbf{A}(t)$,

$S\left(\tilde{\mathbf{p}}, \mathbf{r}, t^{\prime}, t\right)=I_{p} t^{\prime}-\int_{t^{\prime}}^{t}[\dot{\mathbf{p}}(\tau) \cdot \mathbf{r}(\tau)+H(\mathbf{r}(\tau), \mathbf{p}(\tau), \tau] d \tau$,

and

$$
H(\mathbf{r}(\tau), \mathbf{p}(\tau), \tau)=\frac{1}{2}[\mathbf{p}(\tau)+\mathbf{A}(\tau)]^{2}-\frac{C}{\sqrt{\mathbf{r}(\tau) \cdot \mathbf{r}(\tau)}}
$$

One should note that the problem is solved in the length gauge, and Eq. (24) can be obtained from the standard length-gauge Hamiltonian by a partial integration. This issue has been also discussed in detail in Ref. [69].

Following the same procedure as for the SFA, we can now obtain the Coulomb-corrected transition amplitude by applying the saddle-point approximation. By construction, the saddle-point equation on $t^{\prime}$ leads to the condition

$$
\frac{\left[\mathbf{p}_{0}+\mathbf{A}\left(t^{\prime}\right)\right]^{2}}{2}+I_{p}+V\left[\mathbf{r}\left(t^{\prime}\right)\right]=0 .
$$

The Coulomb-corrected transition amplitude is then given by

$$
\begin{aligned}
M\left(\mathbf{p}_{f}\right)= & -i \lim _{t \rightarrow \infty} \int d \tilde{\mathbf{p}}_{0} \int_{\tilde{\mathbf{p}}_{0}}^{\tilde{\mathbf{p}}_{f}(t)} \mathcal{D}^{\prime} \tilde{\mathbf{p}} \int \frac{\mathcal{D} \mathbf{r}}{(2 \pi)^{3}} \sum_{s} \mathcal{C}\left(t_{s}\right) \\
& \times e^{i S\left(\tilde{\mathbf{p}}, \mathbf{r}, t_{s}, t\right)}\left\langle\mathbf{p}_{0}+\mathbf{A}\left(t_{s}\right)\left|H_{I}\left(t_{s}\right)\right| \psi_{0}\right\rangle, \quad(26)
\end{aligned}
$$

where $t_{s}$ are the solutions of the saddle-point equation (25) which are again complex, and we consider the initial momentum $\mathbf{p}_{0}=\mathbf{p}\left(t_{s}\right)$ in Eq. (14). We then use the semi-classical path integral theory developed by Van Vleck and Gutzwiller [66] on the paths $[\mathbf{p}(\tau)]$, and $[\mathbf{r}(\tau)]$. The associated saddle-point equations take the form of classical equations of motion for the trajectories,

$$
\begin{aligned}
\dot{\mathbf{p}}(\tau) & =-\nabla_{\mathbf{r}} V[\mathbf{r}(\tau)], \\
\dot{\mathbf{r}}(\tau) & =\mathbf{p}(\tau)+\mathbf{A}(\tau),
\end{aligned}
$$

whose solutions are $\mathbf{r}_{s}(\tau)$ and $\mathbf{p}_{s}(\tau)$. In terms of such solutions, the Coulomb-corrected transition amplitude finally reads

$$
\begin{aligned}
& M\left(\mathbf{p}_{f}\right) \propto-i \lim _{t \rightarrow \infty} \sum_{s}\left\{\operatorname{det}\left[\frac{\partial \mathbf{p}_{s}(t)}{\partial \mathbf{r}_{s}\left(t_{s}\right)}\right]\right\}^{-1 / 2} \\
& \mathcal{C}\left(t_{s}\right) e^{\left.i S\left(\tilde{\mathbf{p}}_{s}, \mathbf{r}_{s}, t_{s}, t\right)\right)}\left\langle\mathbf{p}_{s}\left(t_{s}\right)+\mathbf{A}\left(t_{s}\right)\left|H_{I}\left(t_{s}\right)\right| \psi_{0}\right\rangle .
\end{aligned}
$$

The sum is over the classical trajectories that begin at position $\mathbf{r}\left(t_{s}\right)$ at time $t_{s}$, and end at momentum $\mathbf{p}(t)$ at time $t \rightarrow \infty$ (sum over multiple solutions with identical $t_{s}$ is implied), and the action $S\left(\tilde{\mathbf{p}}_{s}, \mathbf{r}_{s}, t_{s}, t\right)$ is given by Eq. (22). Throughout, we have considered the principal branch. In practice, $t$ should be taken to be sufficiently long so that the electron is far away from the core.

Due to the presence of the binding potential, the above-stated equation exhibits branch cuts for $\operatorname{Re}\left[\mathbf{r}_{s}(\tau)\right.$. $\left.\mathbf{r}_{s}(\tau)\right]<0$ and $\operatorname{Im}\left[\mathbf{r}_{s}(\tau) \cdot \mathbf{r}_{s}(\tau)\right]=0$. For vanishing transverse momenta, the branching points turn into firstorder poles and this problem is absent (for details on these branch cuts see Ref. [70]). These branch cuts can be avoided if one takes the integration contour along the real time axis once the electron is in the continuum. More specifically, the time integration contour is taken first parallel to the imaginary time axis, and then along the real time axis. This is the procedure taken by most groups when implementing Coulomb-corrected strong-field theories (see, e.g., Refs. [35, 71]).

Furthermore, besides the SFA factor (14) and the tunnel matrix element $\left\langle\mathbf{p}_{s}\left(t_{s}\right)+\mathbf{A}\left(t_{s}\right)\left|H_{I}\left(t_{s}\right)\right| \psi_{0}\right\rangle$, the amplitude now involves the stability $\frac{\partial \mathbf{p}_{s}(t)}{\partial \mathbf{r}_{s}\left(t_{s}\right)}$ of the trajectories. In the limit of vanishing binding potential, the usual SFA is recovered [61]. However, as we will see, this happens in a nontrivial fashion when the degeneracy breaking of trajectories is taken into account. 


\section{Practical implementation}

In order to simplify the calculation and isolate the main effects of the potential on the trajectories, we assume that the electron is ionized by tunneling from the time $t_{s}$ to $t_{s}^{R}=\operatorname{Re} t_{s}$ at a fixed momentum $p_{s}\left(t_{s}\right)$ and then moves to detector with the real time and coordinate according to the classical equations of motion (27) and (28). This is the most widely used assumption for the contour, and has been employed in $[35,43,44]$ (for a review see [69]). Because we assume that the momentum of the electron is fixed during the tunneling ionization, it is a reasonable approximation to neglect the Coulomb potential in Eq. (25), which thus reduces to Eq. (12). The point in space for which the time becomes real is widely known as "the tunnel exit". Physically, this also gives the point in space at which the electron tunnels out of the potential barrier.

The tunneling exit at the time $t_{R}$ is given by

$$
z_{0}=\alpha\left(t_{s}^{R}\right)-\operatorname{Re} \alpha(t)
$$

where $\alpha(t)=\int^{t} \mathbf{A}(\tau) d \tau$ [72]. We use the tunnel exit approximation (30) to split the action into a part inside the barrier,

$$
\tilde{S}_{s}^{\text {in }}\left(\tilde{\mathbf{p}}_{s}, \mathbf{r}_{s}, t_{s}\right)=-\int_{t_{s}}^{t_{s}^{R}} H\left(\mathbf{r}_{s}, \mathbf{p}_{s}, \tau\right) d \tau
$$

with the tunneling trajectory $\mathbf{r}_{s}(t)=\int_{t_{s}}^{t}\left[\mathbf{p}_{s}(\tau)+\mathbf{A}(\tau)\right] d \tau$ [43] and a part outside the barrier,

$$
\tilde{S}_{s}^{\text {out }}\left(\tilde{\mathbf{p}}_{s}, \mathbf{r}_{s}, t_{s}\right)=\int_{t_{s}^{R}}^{T_{p}} d \tau\left[-\dot{\mathbf{p}}_{s}(\tau) \cdot \mathbf{r}_{s}(\tau)-H\left(\mathbf{r}_{s}, \mathbf{p}_{s}, \tau\right)\right]
$$

with the ionization trajectory determined as described in the previous subsection. Note that, in Eq. (31), the term in $\dot{\mathbf{p}}_{s}$ is vanishing as the drift momentum inside the barrier was taken to be constant. Furthermore, real variables outside the barrier will lead to real stability factors, so that phase differences in the continuum stem exclusively from the action. The part inside the barrier is starting from the origin up to the tunnel exit. In practice, because of the singularity of the Coulomb potential at the origin, the tunneling time is shifted along the contour by a very small value, so that the trajectory of the electron begins not exactly at the origin. However, the initial coordinate is still sufficiently close to it so that the transformation below can be used. The same procedure is also performed in Ref. [71]. In this work, we have also considered the same shift for the imaginary part of the tunneling time as in [71], namely $i \omega /\left(2 I_{p}\right)$.

Within the CQSFA, we calculate the stability of the trajectories numerically. In practice, instead of using $\partial \mathbf{p}_{s}(t) / \partial \mathbf{r}_{s}\left(t_{s}\right)$ in Eq. (29) we employ $\partial \mathbf{p}_{s}(t) / \partial \mathbf{p}_{s}\left(t_{s}\right)$. The latter stability factor is of easier implementation, and can be obtained using a Legendre transformation in the transition amplitude (26). Upon this transformation, the action will remain the same as long as the electron starts from the origin. For details on Legendre transformations see, e.g., [77]. The normalization constants in the above-stated equations are such that, in the limit of vanishing binding potential, the SFA transition amplitude is recovered.

Our method is closely related, but different from the existing CCSFA [35, 43, 44] and the Eikonal Volkov approximation [41, 42]. Conceptually, our derivation is based on the Feynman path integral approximation, which is applied to the time-evolution operation in the presence of the Coulomb potential. This is a different starting point from the derivations in [35, 41-44]. The eikonal Volkov approximation starts from a laser-dressed WKB-type approximation and requires small scattering angles. The CCSFA theory takes the Coulomb-free trajectories as a zero-order approximation and accounts for the Coulomb field perturbatively via corrections to the classical action. It is noteworthy that neither method leads to the extra term $\dot{\mathbf{p}}_{s}(\tau) \cdot \mathbf{r}_{s}(\tau)$, which is obtained in our computations. This term is necessary to obtain the correct phases and contrast, and keep the trajectories real in the continuum. Furthermore, we solve the inverse problem, i.e., for a given a final momentum, we compute the initial momentum, while in the CCSFA direct propagation with a shooting method is employed.

\section{RESULTS AND DISCUSSION}

In the results that follow, we use the monochromatic laser field

$$
\mathbf{E}(t)=\hat{\mathbf{z}} E_{0} \sin \omega t
$$

where $E_{0}$ is the peak electric field amplitude. For this type of field, the vector potential of the laser field $\mathbf{A}(t) \propto$ $\cos (\omega t) \hat{\mathbf{z}}$. Hence, whenever there is a crossing for $\mathbf{A}(t)$ there will be a crest for $\mathbf{E}(t)$. In a practical calculation, however, one must consider a pulse of finite duration. In our work, the electron is ionized at the time near $t=0$, i.e., $-\pi / 2<\omega t_{s}<\pi / 2$, and then the ionized electron moves in the presence of the laser field and the Coulomb potential until the time $T_{p}=15.25$ cycles, with $\mathbf{A}\left(T_{p}\right)=0$ and $\left|\mathbf{E}\left(T_{p}\right)\right|=E_{0}$. In the SFA theory, for a given final momentum $\mathbf{p}$, there are two solutions $t_{s}$ of Eq. (12) per cycle of the laser field. In [44], these solutions have been related to Orbits I and II, depending on whether the electron leaves in the same or in the opposite direction to the detector. In the results that follow, we will consider this classification and its extension to the Coulomb-corrected case.

The initial states are taken as the ground state of Hydrogen, i.e., $\psi_{0}(r)=\left\langle\mathbf{r} \mid \psi_{0}\right\rangle=e^{-r} / \sqrt{\pi}$. In this case, the tunnel matrix element in Eq. (29) becomes related to the atomic dipole moment and can be simplified as $\left\langle\mathbf{p}_{s}\left(t_{s}\right)+\mathbf{A}\left(t_{s}\right)\left|-\mathbf{r} \cdot \mathbf{E}\left(t_{s}\right)\right| \psi_{0}\right\rangle \sim E(t) \tilde{p}_{0 z}$, where $\tilde{\mathbf{p}}_{0}=\mathbf{p}_{s}\left(t_{s}\right)+\mathbf{A}\left(t_{s}\right)[67]$. Unless otherwise stated, we will consider $C=1$ in $V(r)$. 


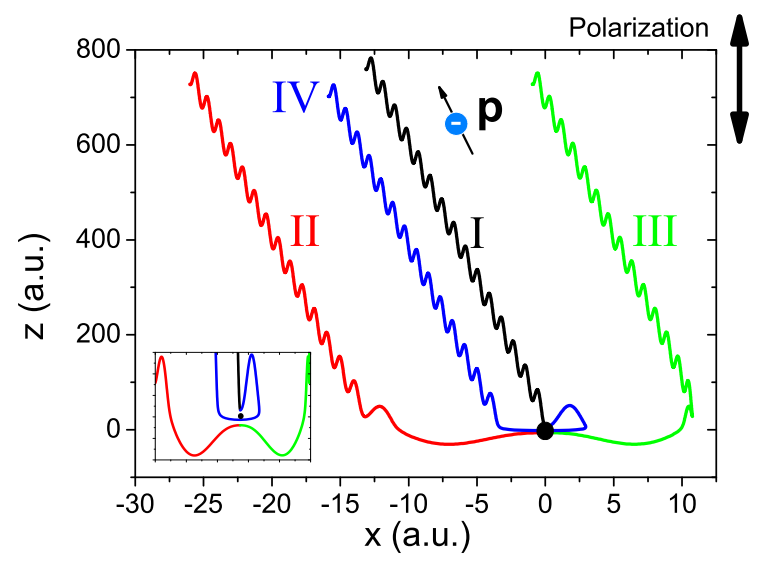

FIG. 1: (Color online) Illustration of the four types of CQSFA trajectories in the $z x$ plane for electrons with fixed final momentum $\mathbf{p}_{f}$, computed using a linearly polarized square pulse which ends at the time $T_{p}=15.25$ cycles, of intensity $I=2 \times 10^{14} \mathrm{~W} / \mathrm{cm}^{2}$ and frequency $\omega=0.057$ a.u., and a Coulomb potential (3) with $C=1$. The ionization potential has been taken as $I_{p}=0.5$ a.u. The black dot denotes the position of the nucleus. The inset shows the region near the core.

\section{A. Coulomb-corrected saddle-point solutions and their physical implications}

In comparison with the SFA, the canonical momentum $\mathbf{p}$ of the electron is time-dependent according to Eq. (27) if the Coulomb interaction is incorporated. Therefore, in the CQSFA theory, the greatest challenge is to solve the saddle-point equations for the tunneling time $t^{\prime}$ and the canonical momentum $\mathbf{p}_{0}$ for any given final momentum $\mathbf{p}_{f}$. One should also bear in mind that, in experiments, the measured photoelectron spectra is a function of the final momentum. Therefore, if, for a given final momentum, the initial conditions for the corresponding electron trajectories could be obtained reversely, it would be easier to understand how these trajectories were influenced by the Coulomb potential.

Figure 1 depicts four types of trajectories in the $z x$ plane for an electron with a given final momentum $\mathbf{p}_{f}$. For trajectories of type I, the tunneling exit $z_{0}>0$, and the electron moves directly towards the detector without returning to its parent core. For the type II and III trajectories, the tunneling exit $z_{0}<0$, meaning that the initial motion carries the electron away from the detector before it turns around and ends up with the stipulated momentum $\mathbf{p}_{f}$. A closer inspection shows that they are similar to Kepler hyperbolae to which a drift motion caused by the field is superimposed [33, 44]. Trajectory types I and II are similar to the so-called "short" and "long" trajectories in the SFA theory. The type III is not found in the SFA and can be observed after the Coulomb potential is considered, which is consistent with earlier work [44]. (a)

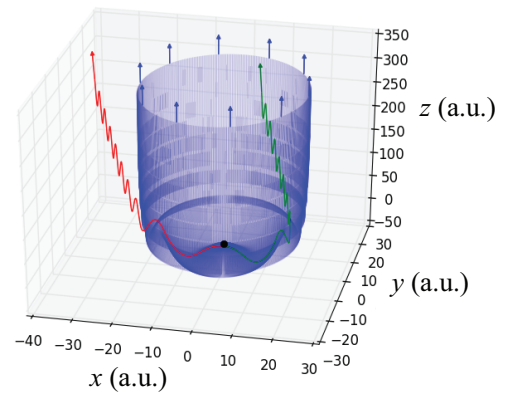

(b)
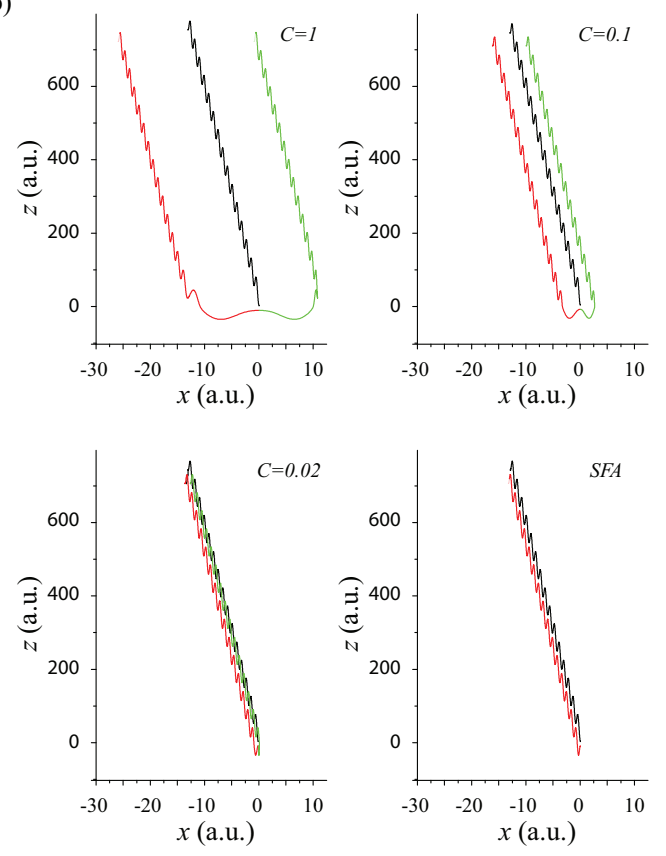

FIG. 2: (Color online) Emergence of the two distinct trajectories II and III. (a) For ionization parallel to the field, the trajectories are part of a torus, respecting the rotational invariance of the system. In the SFA, this torus would contract to a point as momentum conversion then dictates that the initial momentum vanishes. At a finite tilt angle of the final momentum (here $2^{\circ}$ ), however, the torus splits into two distinct solutions, II and III (red and green). (b) As the Coulomb interaction strength $C$ is then reduced, trajectories II and III merge, and remain distinct from trajectory I (here the tilt angle of the final momentum is $1^{\circ}$ ). The remaining parameters are the same as in the previous figure.

As we show in Fig. 2(a), the emergence of the new trajectory is directly related to the momentum nonconservation. It is instructive to start with the case of ionization parallel to the field, where the final momentum $\mathbf{p}_{f}$ is along the polarization direction. On first inspection, the type II and III trajectories are symmetric with respective to the polarization direction. As a matter of fact, trajectories II and III then degenerate into a torus, with a finite initial transverse momentum. In the SFA, the torus contracts onto a single trajectory with a vanishing initial transverse momentum, as dic- 


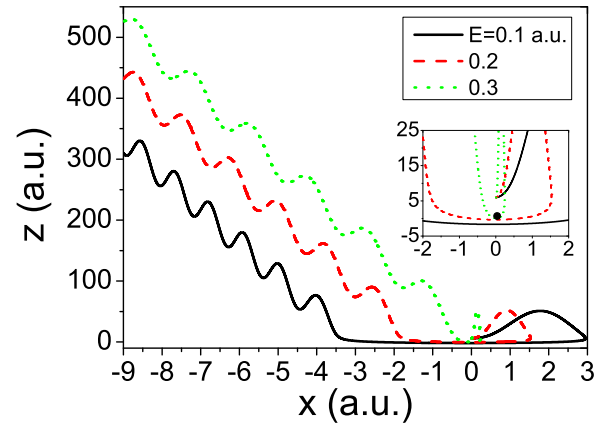

FIG. 3: (Color online) Behavior of type IV orbits for increasing photoelectron energy. The field and atomic parameters are the same as in the previous figures, and the energy is indicated on the upper right corner. A blow-up of the figure shows the trajectories approaching the origin, which is marked by a black dot. The field and atomic parameters are given in Fig. 1.

tated by momentum conservation. At a finite tilt angle, the torus splits in analogy to the Poincaré-Birkhoff scenario in KAM theory, leaving two clearly distinct trajectories. As we change the effective Coulomb interaction strength $C$ from $C=1$ (Hydrogen) to $C=0$, the situation from the SFA is again recovered [panel (b) in Fig. 2].

It is noteworthy that our numerics uncover an additional trajectory type, denoted as IV. Although the tunnel exit points towards the detector, the electron is driven back to the core by the laser field, then goes around the core, and finally moves towards to the detector. With increasing photoelectron final momentum, the shortest distance between the electron and the core decreases. This distance can be smaller than the tunnel exit. In this case, this type of trajectory corresponds to a rescattering event. This behavior is shown in Fig. 3.

Since the contribution of events involving rescattering to the low-energy photoelectron spectra is small, it can be safely neglected, so that we only need to consider the type I-III trajectories. However, it is encouraging to see that rescattering contributions already show up within a framework that is formally tailored for direct ATI, even though they eventually may require a more accurate treatment including rescattering form factors that account for the inherently diffractive, nonclassical nature of these events [19, 24].

\section{B. Photoelectron spectrum}

Based on the trajectories described in the previous subsection we now study the photoelectron spectrum within the CQSFA theory and compare the result with the standard SFA, while taking the ab initio TDSE calculation as a benchmark. The standard SFA is implemented according to Eq. (13). The TDSE has been computed using the freely available software Qprop [76]. In the TDSE

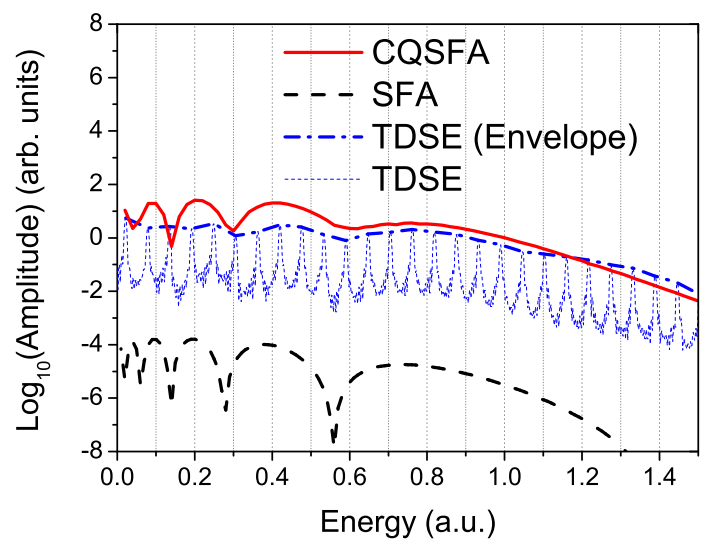

FIG. 4: (Color online) Photoelectron spectra for ionization along the laser polarization direction, with the standard SFA, the Coulomb-corrected CQSFA, and from ab initio TDSE calculation. The blue (dash-dotted) solid curve is the envelope of the TDSE calculation. In order to perform a clearer comparison, the TDSE spectrum has been shifted upwards in around one order of magnitude. The laser-field and atomic parameters are given in Fig. 1.

calculation, the initial state is the ground state of the Coulomb potential (3) which is obtained with imaginary time propagation and is subjected to a long laser pulse $\mathbf{E}(t)=\hat{\mathbf{z}} E_{0} \sin (\omega t) f(t)$ with a trapezoidal profile $f(t)$ (up and down-ramped over 2 cycles, constant over 10 cycles). In practice, the electron wavefunctions are expanded in spherical harmonics and the range of the radial space is from 0 to 6000 a.u. with grid spacing $\Delta r=0.1$ a.u. During the real time propagation, the time step $\Delta t=0.025$ a.u. and the maximal angular momenta is 30 .

The resulting photoelectron spectra in the direction along the laser polarization are shown in Fig. 4. Since the SFA and CQSFA only account for the interference of the electrons ionized in one optical cycle, the spectra correspond to an envelope, without the sharp ATI peaks shown in the $a b$ initio calculation. We therefore also show the envelope of the spectrum from the ab initio method. Clear interference structures are observed in all spectra. A closer inspection shows that the interference contrast in the spectra from the TDSE and the CQSFA theory is much weaker than that from the SFA theory. Moreover, the positions of the interference maxima in the CQSFA spectrum are in a better agreement with the TDSE result than the SFA.

The mechanisms leading to these improvements are explained in the next subsections. For reference, it is useful to inspect how the Coulomb corrections are established when one changes the effective interaction strength $C$, so that for $C=0$ the SFA is recovered. Fig. 5 shows the photoelectron spectra for different values of $C$. 


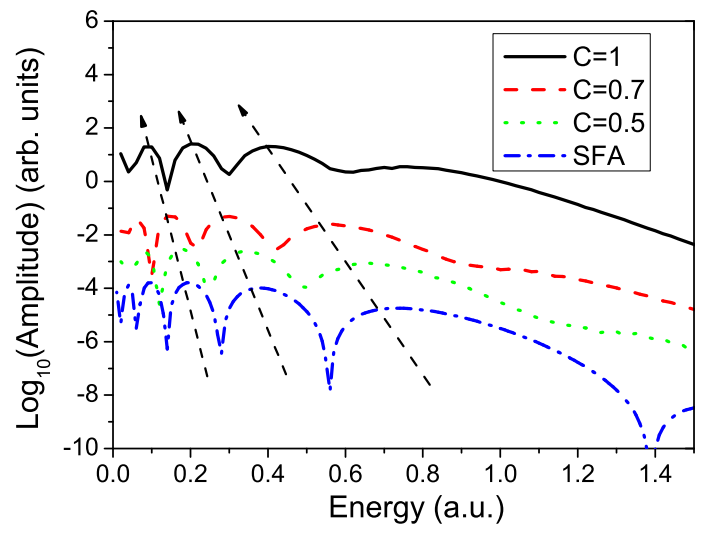

FIG. 5: (Color online) Photoelectron spectra from the CQSFA theory with different strengths $C$ of the rescaled Coulomb potential given by Eq. (3). For $C=0$ the spectrum coincides with that of the SFA theory. The remaining laser-field and atomic parameters are given in Fig. 1.
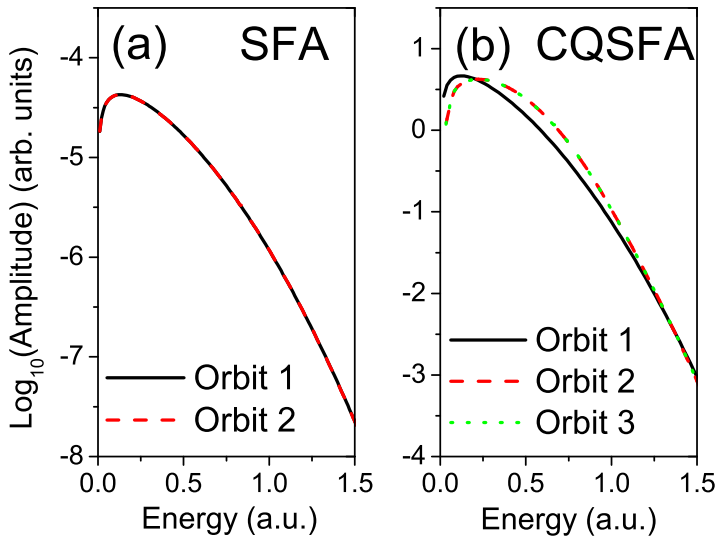

FIG. 6: (Color online) Amplitude of each trajectory type as a function of the photoelectron energy (a) within the SFA theory and (b) within the CQSFA theory. The laser-field and atomic parameters are given in Fig. 1.

\section{Interference contrast}

First, we consider the effect of the Coulomb potential on the interference contrast. According to the discussion above, the type I-III trajectories are dominant for the electrons with low kinetic energy. For the photoelectron spectrum along the laser polarization, type II and III trajectories are symmetric with respect to the polarization direction and have the same phase and amplitude. Therefore, the interference pattern in the spectrum arises from the beating between type I trajectory and type II and III trajectories. In Fig. 6, we present the amplitude related to each orbit as a function of the photoelectron energy with and without Coulomb corrections, respectively.
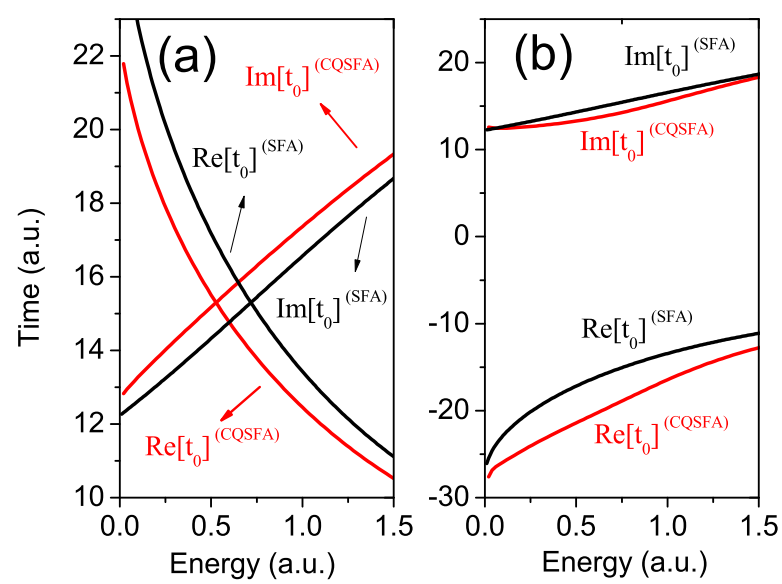

FIG. 7: (Color online) Time of tunneling as a function of the photoelectron energy. (a) for the type I orbit and (b) for the type II orbit. The time is separated into two parts: real part and imaginary part. The black curves are for the SFA calculation and the red (gray) curves are from the CQSFA. The laser-field and atomic parameters are given in Fig. 1.

In the SFA theory, the amplitudes associated with the type I and the type II trajectories are the same. This holds because in the SFA the electron's final momentum is solely determined by the ionization time, which for trajectories I and II are displaced by half a cycle. This means that the absolute values of the electric field, and hence the ionization probability, are the same. Therefore, in the SFA the interference contrast will be maximal. If the Coulomb potential is included, the amplitudes of the type I and II/III trajectories differ slightly. Furthermore, the joint amplitude of the type II and III trajectories exceeds that of type I significantly. All this leads to a much reduced contrast of the interference pattern [? ].

Phenomenological insight into the amplitude difference between the type I and type II/III trajectories can be obtained by considering the tunneling time and initial momentum as a function of the photoelectron energy. The imaginary part $\operatorname{Im}\left[t_{s}\right]$ can be interpreted as the time it takes the electron to tunnel through the potential barrier [68]. The larger $\operatorname{Im}\left[t_{s}\right]$ is, the lower the ionization rate. Fig. 7 exhibits the time of tunneling as a function of the photoelectron energy for the type I orbit and the type II orbit, respectively. For the type I orbit, $\operatorname{Im}\left[t_{s}\right]$ increases when the Coulomb potential is taken into account. In contrast, for type II and III trajectories, $\operatorname{Im}\left[t_{s}\right]$ is smaller in the CQSFA than in the SFA. Therefore, if the Coulomb corrections are present, the amplitude of the type I will be smaller than that of type II. These features are consistent with the changes in $\operatorname{Re}\left[t_{s}\right]$, which, for Orbit I, move towards the field crossing and, for Orbits II/III, is displaced towards the times for which the field amplitude is maximal. This implies that the effective potential barrier will be wider for the former orbits and narrower for the latter. 


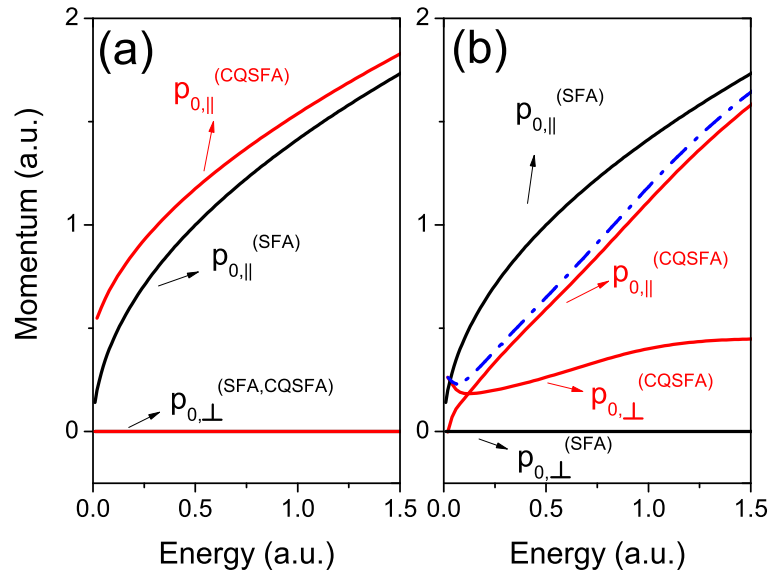

FIG. 8: (Color online) Initial momentum for trajectories of type I (a) and II (b) as a function of the photoelectron energy. The momentum is separated into the parallel and perpendicular components. The blue (dash-dotted) curve in (b) denotes the total momentum from the CQSFA theory. The laser-field and atomic parameters are given in Fig. 1. The subscripts $\|$ and $\perp$ refer to the components of the initial momentum $\mathbf{p}_{0}$ parallel and perpendicular to the laser-field polarization.

According to the Ammosov-Delone-Krainov (ADK) theory [73], these observations can be linked to the initial momentum, with a large momentum translating into a reduced ionization rate. Fig. 8(a) shows that for trajectories of type I, the initial momentum from CQSFA theory is indeed larger than that from the SFA calculation. This can be understood from the fact that the electron needs to compensate the deceleration in the Coulomb potential as it moves towards the detector. In contrast, for type II and III trajectories, the initial parallel momentum from the CQSFA theory is smaller than in the SFA. Although there is a nonvanishing perpendicular momentum from the CQSFA theory, the total initial momentum [see blue curve in Fig. 8(b)] is still lower than that from the SFA theory. This indicates that the electron accelerates significantly due to the interplay of the Coulomb potential and the laser field as it passes near the core. We conclude that the amplitude difference between the different types of trajectories is generally consistent with the phenomenological picture of the ADK theory.

Physically, the above-mentioned behavior can be attributed to the fact that the Coulomb potential decelerates the electron for Orbit I, which hinders ionization. In contrast, for orbits II and III, the Coulomb potential accelerates the electron. Hence, the electron acquires an additional pull, and may escape moving along laser-dressed Kepler hyperbolae.

\section{Positions of interference maxima}

We turn to the positions of interference maxima in the spectra. Fig. 4 shows that the positions of the interfer-

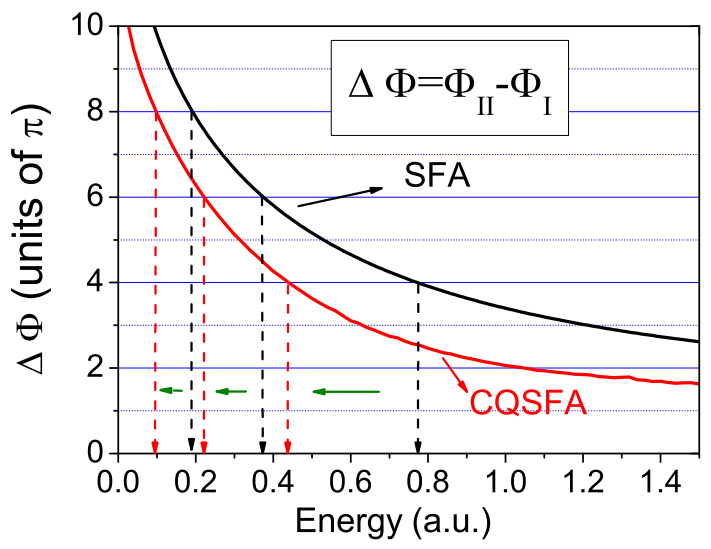

FIG. 9: (Color online) Phase difference $\Delta \Phi=\Phi_{I}-\Phi_{I I}$ (in units of $\pi$ ) between trajectories of type I and II as a function of the photoelectron energy. The arrows denote the positions of the interference maxima, where $\Delta \Phi=2 k \pi, k=1,2, \ldots$. The laser-field and atomic parameters are given in Fig. 1.

ence maxima in the spectra from the CQSFA theory are shifted when compared with the SFA. Since the positions of interference maxima are determined by the phase difference between different types of trajectories we study how this is affected by the Coulomb potential. In Fig. 9, we show the phase difference between the type I and type II trajectories as a function of the photoelectron energy. After considering the Coulomb potential, the dynamical phase difference from the CQSFA becomes smaller than that from the SFA. This can be traced back to the fact that the type II trajectory accumulates a larger negative phase contribution from the Coulomb potential as it passes by the core. Overall, this reduces the phase difference to trajectory I. A similar analysis has been employed in our previous publication [26], in the context of ATI with elliptically polarized fields.

Note that the reduction of the phase difference approaches $2 \pi$, so that neglecting multiples of $2 \pi$ one can also interpret the large shift towards lower energies as a small shift towards larger energies. This ambiguity is resolved when we consider the effect of the continuously rescaled Coulomb potential in Fig. 5, which shows how a large shift towards smaller energies is established as $C$ increases. These results are also consistent with the recent TDSE simulations in [63], and with the outcome of similar Coulomb corrected approaches [35, 43].

\section{Overall ionization amplitude}

Finally, we turn to the overall ionization amplitude of the spectra. As we can see from Fig. 4, the ionization amplitude from the Coulomb-corrected theory is much larger than that from the SFA. Enhanced tunnel ionization is a well-known effect caused by Coulomb corrections to the effective potential barrier. It was first predicted 


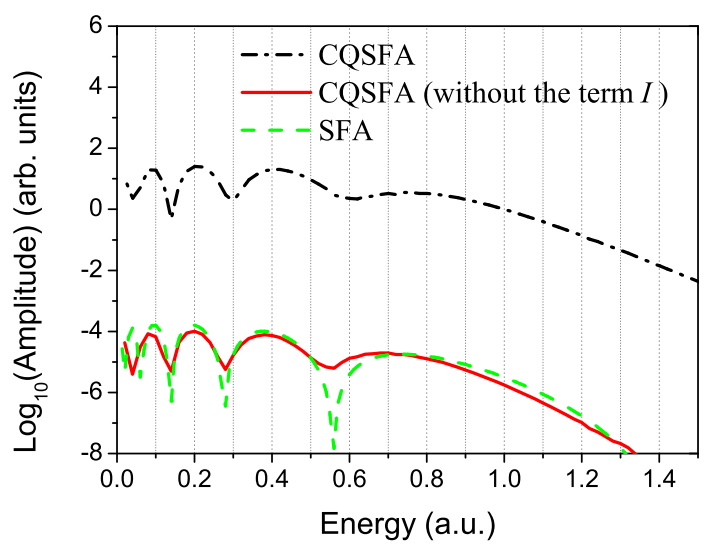

FIG. 10: (Color online) Photoelectron spectra from the CQSFA theory and the SFA theory, respectively. The red (solid) curve is the spectrum from the CQSFA, but without the subbarrier Coulomb term $I=-\int_{t_{s}}^{t_{s}^{R}} V[\mathbf{r}(\tau)] d \tau$. The laserfield and atomic parameters are given in Fig. 1.

in [79] and subsequently observed in several Coulombcorrected strong-field calculations [41, 80]. Furthermore, in the 1980s the Coulomb-induced orders-in-magnitude enhancement of tunnel ionization rates of atoms and positive ions was well documented in experiments [64]. Intuitively, it can be understood that compared to the SFA theory, the barrier for a Coulomb potential is smoother and lower, making it easier for the electron to tunnel through. Within the CQSFA, this enhancement of the ionization amplitude mainly originates from Coulomb term $I=-\int_{t_{s}}^{t_{s}^{R}} V[\mathbf{r}(\tau)] d \tau$ in the sub-barrier action (31). Fig. 10 shows the result if this term is neglected. The overall magnitude of the spectrum is then comparable to the SFA. In the CQSFA this term contributes with a negative imaginary part $\operatorname{Im} I<0$, and thus increases the ionization amplitude.

One should note, however, that the overall probability in the CQSFA simulation depends on the shift of the tunneling time performed in this computation in order to avoid the Coulomb singularity at the origin. The CQSFA simulation shows that the smaller the shift of the tunneling time is, the larger the ionization yield will be. Hence, another method must be applied in order to overcome this problem, in order to make a definitive statement about a quantitative agreement with the TDSE. We have also verified that the contrast and the position of the intra-cycle interference structure are not altered by this parameter.

\section{CONCLUSIONS}

In this work, we develop and use a path-integral formulation to assess the influence of the residual Coulomb potential in above-threshold ionization. We focus on the direct transition amplitude, in which hard collisions with the core are not incorporated. In general, the photoelectron spectra obtained with the Coulomb-corrected method presented in this paper exhibits a superior agreement with the $a b$ initio solution of the time dependent Schrödinger equation to that encountered for the plain strong-field approximation (SFA). This is especially true for the interference substructure in the spectra. As far as the overall ionization probability is concerned, however, a definitive statement cannot yet be made, as it depends on the initial shift in time employed in the sub-barrier part of the contour to avoid the Coulomb singularity. We have however evidence that the presence of the Coulomb potential leads to an increase in the overall ionization yield, which is in line with other Coulomb corrected strong-field theories.

We also perform a systematic analysis of how the Coulomb potential modifies the orbits along which the electron may leave its parent ion and reach the detector. We compare our results with those of the SFA, and make an assessment of how, in the limit of vanishing Coulomb coupling, the SFA is recovered. The present formulation is closely related to the concept of quantum orbits widely employed in semi-analytical strong-field approaches with and without Coulomb corrections.

We have built upon the existing knowledge that the Coulomb potential introduces a richer topology in the electron motion [44], with four distinct sets of orbits, and have related these orbits to those in the SFA in a more systematic way. Throughout, we have employed the same classification as in [44], which specify these four sets as Orbits type I to IV. In particular, we have found that, for electron emission along the polarization axis, due to the rotational symmetry with regard to the field axis, Orbits II and III will be located on a torus. This torus will contract for decreasing Coulomb coupling, until it becomes a point. Physically, this means that, for the SFA, Orbits type II and III will merge into a single, degenerate orbit if the final electron momentum is parallel to the laserfield polarization. For non-vanishing emission angle, the above-mentioned torus will break down, and there will be two discrete solutions. This behavior indicates that, strictly speaking, Orbits type II and III should not be treated independently in an asymptotic expansion when computing photoelectron spectra and momentum distributions. Indeed, a rigorous treatment would require solving the integral around the manifold exactly for a final momentum along the axis, and a uniform approximation for non-vanishing emission angle [74, 75]. This strongly suggests that the cusps observed in $[35,44]$ close to the so-called ATI low-energy structure are related to this effect. It is indeed noteworthy that a very good agreement between the TDSE and the Coulomb-corrected SFA in $[35,44]$ was obtained throughout, except in this region (see also the discussion of this cusp in the review [69]). We have however not studied the above-mentioned cusp systematically.

Furthermore, our results indicate that, if the Coulomb potential is accounted for, the concepts of "direct" and 
"rescattered" electrons are not very clear-cut. These concepts are very clear in the SFA, as there are either hard collisions with the core, or no collisions at all. If the Coulomb corrections are present, however, the Coulomb potential strongly deflects Orbits type IV. These orbits go around the core, and there is a marked decrease in the electron's shortest distance from the origin as the photoelectron momentum increases. For high enough momentum, this distance is located in a region in which the binding potential is dominant. This behavior could be interpreted as a type of recollision, which is absent in the SFA. The amplitude associated with this type of trajectories is however very small and hence not relevant to the computation of ATI spectra in the parameter range of interest.

In addition to that, we have investigated the influence of the Coulomb potential on the ATI spectra, with emphasis on the interference contrast and position of the maxima. This influence has been traced back to particular sets of orbits. First, the contrast in the interference structure decreases, in comparison with the SFA. This happens because, in the SFA, Orbits I and II are equivalent, and displaced by half a cycle, while, if the Coulomb potential is included, this no longer holds. In fact, the Coulomb potential will decelerate the electron if it reaches the continuum along Orbit I, and will accelerate the electron if it is ionized along Orbits II and III. This will lead to an increase in the amplitudes associated with Orbits II and III, and to a decrease in the amplitude related to Orbit I. Furthermore, there is the joint effect of Orbits II and III, which will weaken the fringes. Recently, the influence of Orbit III on interference effects has also been investigated in a different context, namely side-lobes in ATI electron momentum distributions, and it has been found to be significant [78].

The suppression of Orbit I and the enhancement of Orbits II and III has been confirmed by a systematic analysis of the initial momenta and ionization times. For Orbit I, the initial momentum increases when the Coulomb potential is considered, while, for type II/III orbits, the initial momentum decreases. Physically, this means that the Coulomb potential hinders ionization along Orbit I, as the electron will require a larger momentum to escape. For Orbits II/III, the Coulomb potential accelerates the electron after the tunneling ionization, so that a lower escape momentum is required. An increase in the initial momentum for the type I orbits also implies that the electron ionization time has moved away from the field maximum towards the field crossing. This means that the effective potential barrier through which it must tunnel will widen. Hence, there is also an increase in $\operatorname{Im}\left[t_{s}\right]$. In contrast, for Orbits type II/III, the tunneling time moves to the crest of the laser field and thus the effective potential barrier becomes narrower. These observations are consistent with the changes in the real parts $\operatorname{Re}\left[t_{s}\right]$ of these times, as shown in Fig. 7.

Similarly to the results reported in $[35,43,71]$, we also find that there is a phase shift towards lower energies in the interference maxima. In our model, this phase difference occurs due to Coulomb effects in the continuum propagation, while sub-barrier corrections mainly influence the overall yield. In contrast, in $[35,43]$, this phase difference is attributed to sub-barrier corrections instead. While the contour taken by us and the assumption that all variables are real outside the barrier are also employed in $[35,43]$, the term $\dot{\mathbf{p}}_{s}(\tau) \cdot \mathbf{r}_{s}(\tau)$ is absent in their action. Eq. (27) shows that this term is proportional to the gradient of the binding potential. Its value is small for Orbit I, which moves towards the detector directly, while it is large for Orbits II and III, which are deflected by the core before reaching the detector. We have indeed verified that the phase from this term plays an important role in our formulation. Indeed, if this term is removed from the action, there is significant deviation between the TDSE and CQSFA results. The stability factors employed here are also different from those in $[35,43,44]$, but they influence mainly the contrast and not the position of the maxima. They are, however, very important for a good agreement with the full TDSE computations. This term is also absent in [71], in which the Eikonal-Volkov approximation is employed and the phase differences are obtained along propagation by using a complex intermediate coordinate. Since, however, in [71] circularly polarized light is used, it is expected to be vanishingly small as the electron never returns to the core after tunneling ionization. We expect that the present analysis will contribute to a better understanding of cusps and the ATI low-energy structure in the future.

\section{Acknowledgments}

We are grateful to D. Bauer, T. M. Yan, S. Popruzhenko, O. Smirnova, L. Torlina, M. Ivanov, T. Shaaran, J. Biegert, M. Lewenstein and D. B. Milošević for useful discussions. This work was funded by the UK EPSRC (grants EP/J019240/1 and EP/J019585/1), the NNSF of China (Grants Nos. 11204356 and 11474321) and by the Chinese Academy of Sciences overseas study and training program. The data created during this research is openly available [81]. C.F.M.F. would like to thank the Max Born Institute, Berlin and the Chinese Academy of Sciences, Wuhan, for their kind hospitality.
[1] P. Agostini, F. Fabre, G. Mainfray, G. Petite, and N. K. Rahman, Phys. Rev. Lett. 42, 1127 (1979).

[2] W. Becker, F. Grasbon, R. Kopold, D. B. Milošević, G. G. Paulus, and H. Walther, Adv. At. Mol. Opt. Phys.
48, 35 (2002).

[3] K. J. Schafer, B. R. Yang, L. F. DiMauro, and K. C. Kulander, Phys. Rev. Lett. 70, 1599 (1993).

[4] P. B. Corkum, Phys. Rev. Lett. 71, 1994 (1993). 
[5] G. G. Paulus, W. Nicklich, Huale Xu, P. Lambropoulos, and H. Walther, Phys. Rev. Lett. 72, 2851 (1994); A. Gazibegović-Busuladžić, D. B. Milošević, W. Becker, B. Bergues, H. Hultgren, and I. Yu. Kiyan, Phys. Rev. Lett. 104, 103004 (2010).

[6] M. Ferray, A L'Huillier, X. F. Li, L. A. Lompré, G. Mainfray, and C. Manus, J. Phys. B 21, L31 (1988); A. D. Shiner, B. E. Schmidt, C. Trallero-Herrero, H. J. Wörner, S. Patchkovskii, P. B. Corkum, J-C. Kieffer, F. Légaré, and D. M. Villeneuve, Nature Phys. 7, 464 (2011).

[7] B. Walker, B. Sheehy, L. F. DiMauro, P. Agostini, K. J. Schafer, and K. C. Kulander, Phys. Rev. Lett. 73, 1227, (1994).

[8] M. Meckel, D. Comtois, D. Zeidler, A. Staudte, D. Pavičić, H. C. Bandulet, H. Pépin, J. C. Kieffer, R. Dörner, D. M. Villeneuve, and P. B. Corkum, Science 320, 1478 (2008).

[9] H. P. Kang et al., Phys. Rev. Lett. 104, 203001 (2010).

[10] S. Haessler et al., Nat. Phys. 6, 200 (2010).

[11] S. Baker et al., Science 312, 424 (2006).

[12] J. Itatani et al., Nature (London) 432, 867 (2004).

[13] C. Figueira de Morisson Faria and X. Liu, J. Mod. Opt. 58, 1076 (2011).

[14] W. Becker, X. Liu, P. J. Ho, and J. H. Eberly, Rev. Mod. Phys. 84, 1011 (2012).

[15] C. Figueira de Morisson Faria, T. Shaaran and M. T. Nygren, Phys. Rev. A 86, 053405 (2012).

[16] X. L. Hao, J. Chen, W. D. Li, B. B. Wang, X. D. Wang, and W. Becker, Phys. Rev. Lett. 112, 073002 (2014).

[17] J. Javanainen, J. H. Eberly, and Q. C. Su, Phys. Rev. A 38, 3430 (1988); R. Grobe and J. H. Eberly, ibid. 48, 4664 (1993).

[18] M. Lewenstein, P. Salières, and A. L'Huillier, Phys. Rev. A 52, 4747 (1995).

[19] C. Figueira de Morisson Faria, H. Schomerus, and W. Becker, Phys. Rev. A 66, 043413 (2002).

[20] R. Kopold, W. Becker, and M. Kleber, Opt. Commun. 179, 39 (2000)

[21] F. Lindner et al,Phys. Rev. Lett. 95, 040401 (2005).

[22] X. H. Xie et al., Phys. Rev. Lett. 108, 193004 (2012).

[23] X. B. Bian and A. D. Bandrauk, Phys. Rev. Lett. 113, 193901 (2014).

[24] C. Figueira de Morisson Faria, H. Schomerus, X. Liu, and W. Becker, Phys. Rev. A 69, 043405 (2004).

[25] M. Busuladžic̀ et al., Phys. Rev. A 78, 033412 (2008).

[26] X.-Y. Lai and C. Figueira de Morisson Faria, Phys. Rev. A 88, 013406 (2013).

[27] L. V. Keldysh, Zh. Eksp. Teor. Fiz. 47, 1945 (1964) [Sov. Phys. JETP 20, 1307 (1965)].

[28] R. Moshammer, J. Ullrich, B. Feuerstein, D. Fischer, A. Dorn, C. D. Schröter, J. R. Crespo Lopez-Urrutia, C. Hoehr, H. Rottke, C. Trump, M. Wittmann, G. Korn, and W. Sandner, Phys. Rev. Lett. 91, 113002 (2003).

[29] C. I. Blaga et al., Nature Phys. 5, 335 (2009).

[30] W. Quan et al., Phys. Rev. Lett. 103, 093001 (2009).

[31] A. Rudenko, K. Zrost, C. D. Schröter, V. L. B. de Jesus, B. Feuerstein, R. Moshammer and J. Ullrich, J. Phys. B 37, L407 (2004).

[32] Z. J. Chen, T. Morishita, A.-T. Le, M. Wickenhauser, X. M. Tong, and C. D. Lin, Phys. Rev. A 74, 053405 (2006)

[33] D. G. Arbó, S. Yoshida, E. Persson, K. I. Dimitriou, and J. Burgdörfer, Phys. Rev. Lett. 96, 143003 (2006).

[34] D. G. Arbó, E. Persson, K. I. Dimitriou, and J. Burgdörfer, Phys. Rev. A 78, 013406 (2008).

[35] T. M. Yan and D. Bauer, Phys. Rev. A 86, 053403 (2012).
[36] M. Bashkansky, P. H. Bucksbaum, and D. W. Schumacher, Phys. Rev. Lett. 60, 2458 (1988).

[37] S. V. Popruzhenko, G.G. Paulus and D. Bauer, Phys. Rev. A 77, 053409 (2008).

[38] G. Duchateau, E. Cormier, and R. Gayet, Phys. Rev. A 66, 023412 (2002); G. Duchateau, E. Cormier, H. Bachau, and R. Gayet, J. Mod. Opt. 50, 331 (2003).

[39] D. B. Milosevic and F. Ehlotzky, Phys. Rev. A 58, 3124 (1998).

[40] D. G. Arbó, J. Phys. B 47, 204008 (2014).

[41] O. Smirnova, M. Spanner, and M. Ivanov, J. Phys. B 39, S307 (2006), ibid. S323 (2006).

[42] O. Smirnova, M. Spanner, and M. Ivanov, Phys. Rev. A 77, 033407 (2008)

[43] S.V. Popruzhenko and D. Bauer, J. Mod. Opt. 55, 2573 (2008).

[44] T. M. Yan, S. V. Popruzhenko, M. J. J. Vrakking, and D. Bauer, Phys. Rev. Lett. 105, 253002 (2010).

[45] M. Li et al., Phys. Rev. Lett. 112, 113002 (2014).

[46] G. van de Sand and J.-M. Rost, Phys. Rev. Lett. 83, 524 (1999).

[47] C. Zagoya, C.-M. Goletz, F. Grossmann, and J.M. Rost, Phys. Rev. A 85, 041401(R) (2012).

[48] C. Zagoya, C.-M. Goletz, F. Grossmann, and J.M. Rost, New J. Phys. 14, 093050 (2012).

[49] C. Zagoya, J. Wu, M. Ronto, D. V. Shalashilin, C. Figueira de Morisson Faria, New J. Phys. 16, 103040 (2014).

[50] J. Guo, X. S. Liu, and Shih-I Chu, Phys. Rev. A 82, $023402(2010)$.

[51] A. Kirrander and D.V. Shalashilin, Phys. Rev. A 84, 033406 (2011).

[52] C. Symonds, J. Wu, M. Ronto, C. Zagoya, C. Figueira de Morisson Faria, and D. V. Shalashilin, Phys. Rev. A 91, 023427 (2015).

[53] L. Torlina and O. Smirnova, Phys. Rev. A 86, 043408 (2012); L. Torlina, F. Morales, H. G. Muller and O. Smirnova, J Phys B 47, 204021 (2014).

[54] L. Torlina, M. Ivanov, Z. B. Walters, and O. Smirnova, Phys. Rev. A 86, 043409 (2012).

[55] F. H. M. Faisal, J. Phys. B 6, L89 (1973).

[56] H. R. Reiss, Phys. Rev. A, 22, 1786 (1980).

[57] W. Becker, A. Lohr, M. Kleber and M. Lewenstein, Phys. Rev. A 56, 645 (1997).

[58] A. Fring, V. Kostrykin and R. Schrader, J. Phys. B 29, 5651 (1996).

[59] O. Smirnova, M. Spanner and M. Ivanov, J. Mod. Opt. 52, 165 (2005); ibid. 54, 1019 (2007).

[60] P. Salières, B. Carré, L. Le Déroff, F. Grasbon, G.G. Paulus, H. Walther, R. Kopold, W. Becker, D.B. Milošević , A. Sanpera, M. Lewenstein, Science 292, 902 (2001).

[61] D. B. Milošević, J. Math. Phys. 54, 042101 (2013).

[62] I. Barth and O. Smirnova, Phys. Rev. A 84, 063415 (2011).

[63] D. G. Arbó, K. L. Ishikawa, K. Schiessl, E. Persson, and J. Burgdörfer, Phys. Rev. A 81, 021403(R) (2010).

[64] S. L. Chin, C. Rolland, P. B. Corkum, and P. Kelly, Phys. Rev. Lett. 61, 153 (1988).

[65] H. Kleinert, Path integrals in quantum mechanics, statistics, polymer physics, and financial markets, (World Scientific, 2009).

[66] K. G. Kay, Phys. Rev. A, 88, 012122, (2013).

[67] D. B. Milošević, G. G. Paulus, D. Bauer and W. Becker, J. Phys. B 39, R203 (2006). 
[68] E. H. Hauge, and J. A. Stovneng, Rev. Mod. Phys. 59, 917 (1989).

[69] S. V. Popruzhenko, J. Phys. B 47, 204001 (2014).

[70] S. V. Popruzhenko, Zh. Eksp. Teor. Fiz. 145, 664 (2014) [JETP Phys. 118, 580 (2014)].

[71] Lisa Torlina, Jivesh Kaushal, and Olga Smirnova, Phys. Rev. A 88, 053403 (2013).

[72] A. M. Perelomov, V. S. Popov and M. V. Terentev, Sov. Phys. JETP 24, 207 (1967) [Zh. Eksp. Theoret. Fiz. (U.S.S.R.) 51, 309 (1966)].

[73] M. V. Ammosov, N. B. Delone and V. P. Krainov, Sov. Phys. JETP 64, 1191 (1986) [Zh. Eksp. Theoret. Fiz. (U.S.S.R.) 91, 2008 (1986)].

[74] S. Tomsovic, M. Grinberg and D. Ullmo, Phys. Rev. Lett. 75, 4346 (1995).

[75] D. Ullmo, M. Grinberg, and S. Tomsovic, Phys. Rev. E 54, 136 (1996).

[76] D. Bauer and P. Koval, Comp. Phys. Comm. 174, 396 (2006); see also the website www.qprop.de for more documentation.

[77] H. Goldstein, C. P. Poole and J. L. Safko, Classical Me- chanics (Addison Wesley, 2002)

[78] T.-M. Yan, S.V. Popruzhenko and D. Bauer, in: Progress in Ultrafast Intense Laser Science IX, K. Yamanouchi, K. Midorikawa (Eds.) (Springer, Heidelberg, 2013)

[79] A. M. Perelomov and V. S. Popov, Zh. Eksp. Teor. Fiz. 52, 514 (1967)[Sov. Phys. JETP 25, 336 (1967)]; see also the review article V. S. Popov, Phys. Usp. 47, 855 (2004).

[80] S. V. Popruzhenko, V. D. Mur, V. S. Popov and D. Bauer, Phys. Rev. Lett. 101, 193003 (2008).

[81] The data is openly available at http://dx.doi.org/10.17635/lancaster/researchdata/8

[82] The phase $\exp [-i \mathbf{A}(t) \cdot \mathbf{r}]$ associated with the gauge transformation will cancel out that existent in the Volkov state.

[83] In principle, the Poincaré-Birkhoff type scenario has implications for the saddle-point treatment $[74,75]$, in analogy to the uniform approximations needed close to orbit bifurcation scenarios [19, 24], but as we do not encounter any divergence and in view of providing a simple picture, we did not find it necessary to employ this here. 